\title{
The Massive Star Population at the Center of the Milky Way
}

\author{
Francisco Najarro ${ }^{1}$, Diego de la Fuente ${ }^{1}$, Tom R. Geballe ${ }^{2}$, \\ Don F. Figer ${ }^{3}$ and D. John Hillier ${ }^{4}$ \\ ${ }^{1}$ Centro de Astrobiología (CSIC/INTA), ctra. de Ajalvir km. 4, 28850 Torrejón de Ardoz, \\ Madrid, Spain \\ ${ }^{2}$ Gemini Observatory, 670 N. A'ohoku Place, Hilo, HI 96720, USA \\ ${ }^{3}$ Center for Detectors, Rochester Institute of Technology, 54 Lomb Memorial Drive, Rochester, \\ NY 14623, USA \\ ${ }^{4}$ Department of Physics and Astronomy, University of Pittsburgh, 3941 O'Hara Street, \\ Pittsburgh, PA 15260
}

\begin{abstract}
Recent detection of a large number of apparently isolated massive stars within the inner $80 \mathrm{pc}$ of the Galactic Center has raised fundamental questions regarding massive star formation in a such a dense and harsh environment. Are these isolated stars the results of tidal interactions between clusters, are they escapees from a disrupted cluster, or do they represent a new mode of massive star formation in isolation? Noting that most of the isolated massive stars have spectral analogs in the Quintuplet Cluster, we have undertaken a combined analysis of the infrared spectra of both selected Quintuplet stars and the isolated objects using Gemini North spectroscopy. We present preliminary results, aiming at $\alpha$-elements vs iron abundances, stellar properties, ages and radial velocities which will differentiate the top-heavy and star-formation scenarios.
\end{abstract}

Keywords. stars: early-type -stars: mass loss - stars: winds - stars: abundances - Galaxy: center - infrared: stars

\section{Massive stars in clusters and isolation at the Galactic Center. Star formation scenario}

The Galactic Center (GC), hosting three of the most massive young clusters in the Local Group (Central, Arches and Quintuplet), is a unique laboratory to investigate massive stars and massive star formation. Given the intense radiation fields and extreme stellar densities present in the GC, one may question if star formation occurs in the same manner as seen in the giant molecular clouds elsewhere in the Galaxy. Recent results have revealed the presence of a large number of isolated massive stars (Mauerhan et al. 2010a,b) at the GC which is comparable to the massive star population of each of the clusters (Figer et al. 1999, 2002). Such detection of apparently isolated massive stars in this region has raised a further fundamental issue - whether these massive field stars are results of tidal interactions among clusters, are escapees from a disrupted cluster, or represent a new mode of massive star formation in isolation (Dong et al. 2014). The first option has been investigated (Habibi et al. 2014) based on the spectral analogy of these "field" stars with those in the Quintuplet and Arches clusters suggesting that they could have been physically associated with the clusters. Indeed, following the numerical dynamical simulations from Harfst et al. (2010), and including the effects of stellar evolution and the orbit of the Arches cluster in the Galactic Center potential, Habibi et al. (2014) models were able to account for $\sim 60 \%$ of the isolated sources within the 

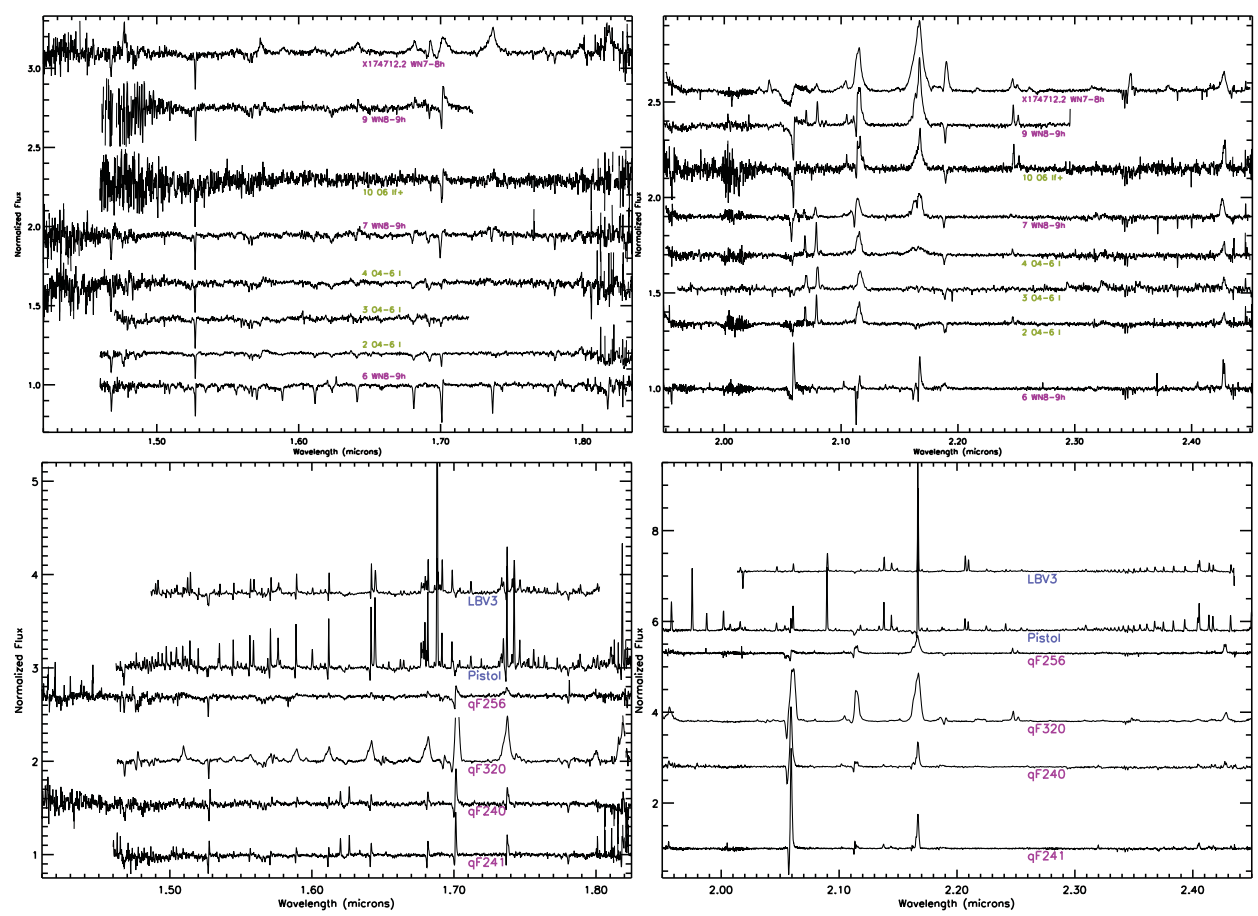

Figure 1. A representative sample of H- and K-band spectra isolated (top) and Quintuplet (bottom) massive stars, showing the diversity of Wolf-Rayet (magenta), OIf (green) and LBVs (blue) stellar population.

central $100 \mathrm{pc}$ as sources drifted away from the center of the clusters. On the other hand, radial velocity measurements of eight objects in the vicinity of the Arches cluster (Dong et al. 2014) and comparisons with those of the cluster, nearby ionized gas and molecular clouds suggest that two of them could have been associated with the cluster while other two likely formed in isolation. Also, from radial velocity studies of WR102ka and a deep integral-field spectroscopy survey of its surroundings Oskinova et al. (2013) concluded that the star likely formed in isolation. However, we note that radial velocity estimates of these objects (mainly OIf + and WNh) may be subject to high uncertainties, as the spectral lines utilized in these studies are severely contaminated by the stellar winds (see below). Thus, further detailed evidence for or against these scenarios is still lacking and awaits precise proper motion measurements (currently underway) providing $3-\mathrm{D}$ velocities of the sources relative to the clusters.

A major step to differentiate among the above scenarios can be achieved through spectroscopic studies of the isolated sources, yielding stellar properties, ages and abundances. Comparison of the results of the quantitative model-atmosphere analysis to theoretical isochrones will allow us to determine if these stars were born in single co-eval cold molecular cloud event or formed over an extended (e.g., 1-10 Myr) period. Obtaining metal abundances from these "field" objects is crucial, not only to understand the metal enrichment history of the GC, but also to test whether these isolated stars have followed a metal-enrichment scenario different than those in the GC clusters. The Quintuplet and Arches clusters provide the stellar reference sources to perform such studies. At its current evolutionary phase (age $\sim 4 \mathrm{Myr}$, Figer et al. 1999) the massive members of the Quintuplet Cluster are currently WN9-10h (plus a WN6), weak lined WC9, OIf+ stars and LBVs (Figer et al. 1999; Liermann et al. 2009), while the massive population of the 

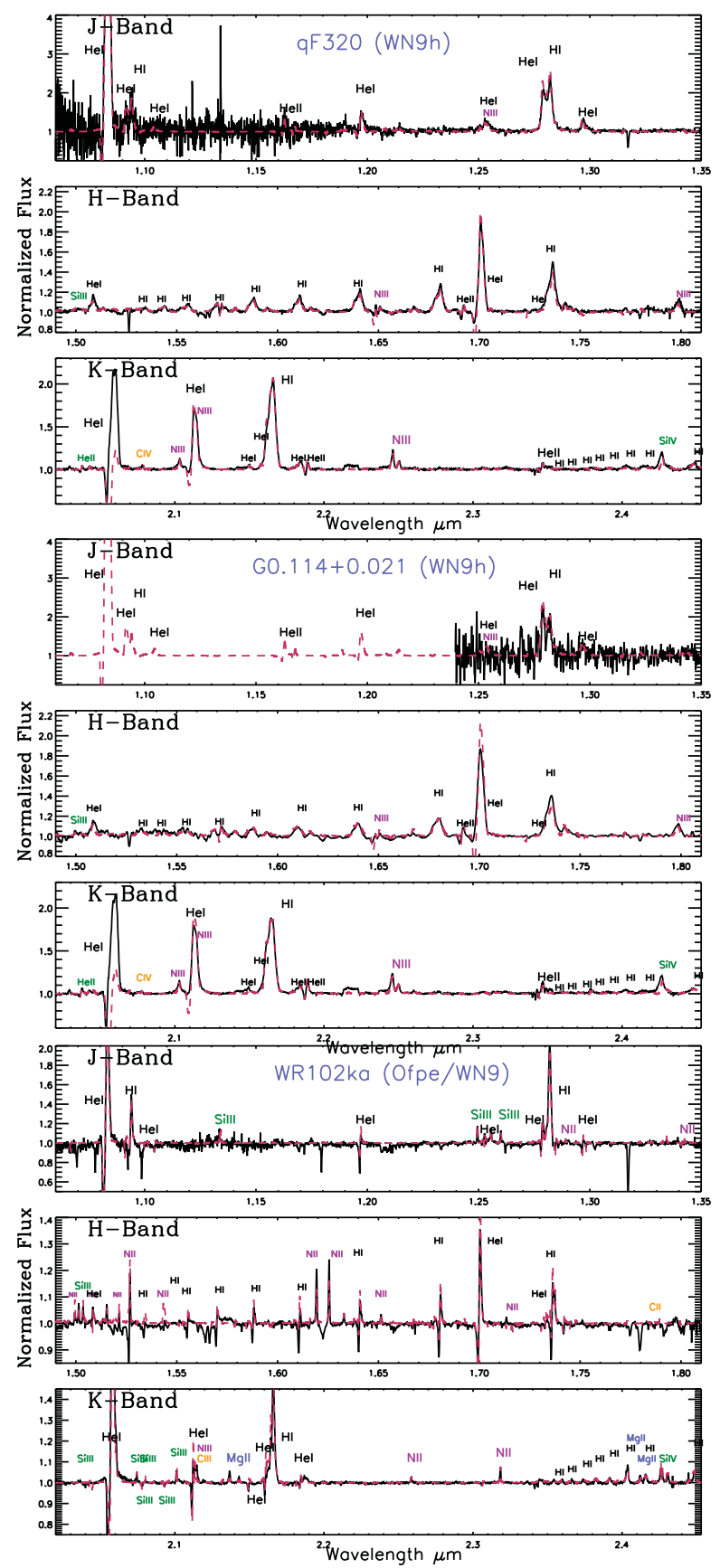

Figure 2. Preliminary fits to the NIR spectra of the Quintuplet star qF320 (WN9h) and the isolated massive stars G0114+0.021 WN8-9h and WR102ka (Ofpe/WN9).

younger Arches cluster is dominated by WN8-9h and OIf + stars (Figer et al. 2002; Najarro et al. 2004; Martins et al. 2008). These spectral types basically encompass all the isolated evolved massive stars identified from recent follow-up spectroscopic observations of $\mathrm{Pa} \alpha$ emission objects in the GC (Mauerhan et al. 2010a,b) which show quite similar 
spectral morphology to those present in the Quintuplet Cluster and some of the brightest Arches members.

Another current hot-topic is whether the IMFs of massive star clusters are top-heavy. In such occurrences the larger number of type II supernovae produce enhanced yields of $\alpha$-elements, resulting in an increase of $\alpha$-element vs Fe (the main suppliers of iron are type Ia SNe). Najarro et al. $(2004,2009)$ have shown that quantitative NIR spectroscopy of high-mass stars may provide estimates of both absolute abundances and abundance ratios, telling us about the global integrated enrichment history up to the present, placing constraints on models of galactic chemical evolution, and acting as clocks by which chemical evolution can be measured. Abundance analyses may thus help to distinguish between top-heavy and standard star formation in the region.

Finally, the presence of three LBVs in the Quintuplet cluster, well in excess of the empirical Humphreys-Davidson limit implying ZAMS masses $>100 M_{\odot}$, requires further investigation. In theory the existence of such stars is problematic, and thus it is important to test for multiplicity in order to address topics such as the high mass cutoff to the formation of massive stars.

\section{Observations and ongoing analysis}

Bearing the above scientific challenges in mind, we started in 2010 an observing program at GEMINI North (currently ongoing) which has been obtaining high S/N, mediumresolution spectra of the most massive stars in the Quintuplet as well as isolated massive stars in the inner GC. So far, around 20 massive stars in the Quintuplet cluster and GC inner region have been observed since 2011 with GEMINI NIFS and GNIRS near infrared spectrographs in the $\mathrm{H}$ and $\mathrm{K}$ bands at medium-resolution ( $\mathrm{R}$ 5000). The brightest targets were also secured at the shorter X and J Bands. A representative sample, consisting in LBVs, OIfs, and WNL stars is presented in Fig. 1. We expect to complete our sample by Aug-Sep 2015. We are currently in the process of modeling the early-type spectra with the CMFGEN code (Hillier \& Miller 1998), to obtain physical and chemical properties. Figure 2 displays preliminary fits to three stars of our sample (qF320, a WN9h star in the Quintuplet cluster, G0.114+0.021, a heavily reddened WN8-9h star close to the Arches and WR102ka, a Ofpe/WN9 object relatively far from the three clusters). At this stage, we may anticipate below some results from our ongoing analysis:

- We obtain a clear $\alpha$-element enrichment from the analysis of the Quintuplet WNh stars, consistent with the results derived for the LBVs (Najarro et al. 2009) which denoted a clearly enhanced $\alpha / \mathrm{Fe}=2$ ratio with respect to solar.

- Stellar abundances of the isolated objects seem to show a similar trend on average, with the presence of even higher $\alpha$-element enrichment in some cases.

- Our new high S/N spectroscopic J, H and K data provide important diagnostic lines (NII-III, SiII-IV, CII-IV, etc.), which are crucial not only for abundance determinations but also to constrain stellar properties. As an example, previously found uncertainties in $T_{\text {eff }}( \pm 6000 \mathrm{~K})$ for the Ofpe/WN9 objects at the GC (Najarro et al. 1997; Martins et al. $2007)$ due to the lack of He II lines in the spectra, are drastically reduced $( \pm 1000 \mathrm{~K})$ by making use of the NII/NIII and SiIII/SiIV ionization equilibria (e.g. WR102ka).

- When available the $\mathrm{HI}$ and $\mathrm{He}$ lines at $\mathrm{P} \beta$ provide an excellent $\mathrm{He} / \mathrm{H}$ ratio diagnostic, allowing much more accurate He abundance determinations than those performed by means of K-Band spectra. Simple blue (HeI) to red (HI) peak ratios may be used (see qF320 and G0.114+0.021 P $\beta$ complex in Fig. 2)

- Radial velocity estimates, if obtained for OIf + and WNh stars, require detailed modeling of the observed spectra (Figer et al. 2004). For the OIf+ stars, the HeII absorption 
lines, which are decent diagnostics for $\mathrm{O}$ V and for some O I stars with weak-to-moderate stellar winds start to be filled by the stellar wind, producing an effective blue-shift as high as $80-90 \mathrm{~km} \mathrm{~s}^{-1}$. This may have important consequences when associating the radial velocities of these objects with the nearby gas and clusters. Further, even quantitative modeling may suffer from high uncertainties. Our preliminary fits to WR 102ka making use of the full $\mathrm{J}, \mathrm{H}$ and $\mathrm{K}$ band spectra reveal a radial velocity of $\sim 100 \mathrm{~km} \mathrm{~s}^{-1}$. This value differs significantly from the $60 \mathrm{~km} \mathrm{~s}^{-1}$ obtained by Oskinova et al. (2013) by means of only K-band spectra and with a slightly (25\%) lower spectral resolution.

\section{Acknowledgements}

F. N. and D.dF. acknowledge grants AYA2010-21697-C05-01 and FIS2012-39162-C0601 and ESP2013-47809-C3-1-R.

\section{References}

Dong, H., Mauerhan, J., Morris, M. R., Wang, Q. D., \& Cotera, A. 2014, in L. O. Sjouwerman, C. C. Lang, \& J. Ott (eds.), IAU Symposium, Vol. 303 of IAU Symposium, pp 230-234

Figer, D. F., McLean, I. S., \& Morris, M. 1999, ApJ 514, 202

Figer, D. F., Najarro, F., Gilmore, D., et al. 2002, ApJ 581, 258

Figer, D. F., Najarro, F., \& Kudritzki, R. P. 2004, ApJ (Letters) 610, L109

Habibi, M., Stolte, A., \& Harfst, S. 2014, A\&A 566, A6

Harfst, S., Portegies Zwart, S., \& Stolte, A. 2010, MNRAS 409, 628

Hillier, D. J. \& Miller, D. L. 1998, ApJ 496, 407

Liermann, A., Hamann, W.-R., \& Oskinova, L. M. 2009, A\&A 494, 1137

Martins, F., Genzel, R., Hillier, D. J., et al. 2007, A\&A 468, 233

Martins, F., Hillier, D. J., Paumard, T., et al. 2008, A\& A 478, 219

Mauerhan, J. C., Cotera, A., Dong, H., et al. 2010a, ApJ 725, 188

Mauerhan, J. C., Muno, M. P., Morris, M. R., Stolovy, S. R., \& Cotera, A. 2010b, ApJ 710, 706

Najarro, F., Figer, D. F., Hillier, D. J., Geballe, T. R., \& Kudritzki, R. P. 2009, ApJ 691, 1816

Najarro, F., Figer, D. F., Hillier, D. J., \& Kudritzki, R. P. 2004, ApJ (Letters) 611, L105

Najarro, F., Krabbe, A., Genzel, R., et al. 1997, A\&̈A 325, 700

Oskinova, L. M., Steinke, M., Hamann, W.-R., et al. 2013, MNRAS 436, 3357

\section{Discussion}

NiEva: Comment. I don't think that the Sun is an appropriate reference for chemical abundances in massive stars. Since a few years we have a reference derived from massive stars which is able to reproduce better the CNO nuclear path than the Asplund's values. Ref: Nieva \& Przybilla (2012), confirmed by Irrgang et al. (in prep) for a larger sample. The stellar evolution models could also take this new reference into account.

NAJARRO: I agree, but so far we have to compare to evolutionary models and their assumed abundances. It would be nice to have models for massive stars with twice $\alpha$ and solar Fe group.

LoBel: You indicate that the Pistol star could be binary (Martayan, in prep.). This is a very important result since the number of LBVs detected in binary systems is growing (four are known). It could hint at a link between the LBV phenomenon and binarity.

NAJARRo: There could be, but there are LBVs like P Cyg or AG Car which have proven not to be binaries. On the other hand I recall the LBV close to SGR1806-20 to be a spectroscopic binary. 